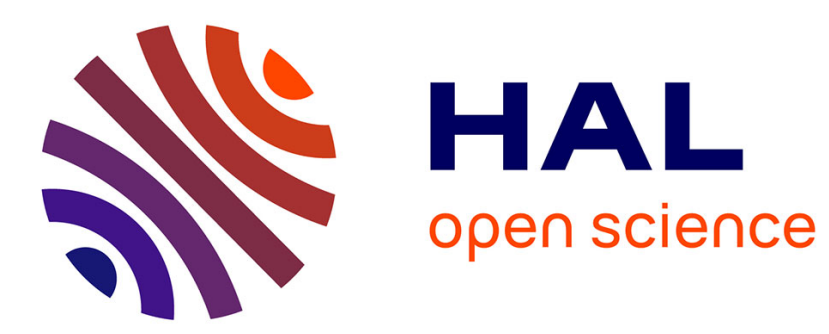

\title{
L'arc expérientiel de l'adolescence : esquive, combine, embrouille, carapace et étincelle
}

Marc Breviglieri

\section{To cite this version:}

Marc Breviglieri. L'arc expérientiel de l'adolescence : esquive, combine, embrouille, carapace et étincelle . Education et Sociétés: Revue internationale de sociologie de l'éducation, 2007, vol. 1 (n¹9), pp. 99-113. hal-01578030v2

\section{HAL Id: hal-01578030 \\ https://hal.science/hal-01578030v2}

Submitted on 22 Jun 2021

HAL is a multi-disciplinary open access archive for the deposit and dissemination of scientific research documents, whether they are published or not. The documents may come from teaching and research institutions in France or abroad, or from public or private research centers.
L'archive ouverte pluridisciplinaire $\mathbf{H A L}$, est destinée au dépôt et à la diffusion de documents scientifiques de niveau recherche, publiés ou non, émanant des établissements d'enseignement et de recherche français ou étrangers, des laboratoires publics ou privés. 


\title{
L'arc expérientiel de l'adolescence : esquive, combine, embrouille, carapace et étincelle...
}

\author{
MARC BREVIGLIERI \\ IUT de Paris V \\ Groupe de Sociologie Politique et Morale, EHESS, CNRS \\ 10, rue Monsieur Le Prince, 75006 Paris
}

C

e texte entend s'installer dans le monde de perception de l'adolescence. Il

part de l'idée qu'au sortir de l'enfance, l'adolescent est directement concerné par une multiplication inédite et progressive d'épreuves en public. Son angle de vue change: le domaine public rayonne pour lui d'une visibilité nouvelle et devient prégnant dans son existence. Vivre l'expérience du domaine public, c'est éprouver les conditions pragmatiques d'un certain ordonnancement des choses du monde et d'une certaine scène d'apparition (Arendt 1983). Cela correspond aussi à la conscience nouvelle d'un temps commun : un temps de vie et d'ouvrage, traversant une histoire collective, qui voit s'élargir les potentialités de l'agir. Autrement dit, c'est tant un sens élargi du devoir qu'une impression conséquente de puissance qui se dilatent à l'adolescence.

Une ligne de tension marque l'écart entre un monde proximal familier, où baigne encore largement l'enfance, et le domaine public, celui des espaces probatoires où parvient l'adolescent avec le désir, la crainte et le devoir d'y affirmer des qualités en propre. La spatialité de l'enfant est pour commencer essentiellement une "spatialité d'attache" où s'épanouit un rapport fondé sur la manipulation du monde familier (Merleau-Ponty 1994). Elle contraste avec l'espace projectif et représentationnel du domaine public dont la dimension est objective et moralisée. À l'adolescence, la jeune personne suit un itinéraire qui tend à la déplacer d'une spatialité d'attache à une spatialité d'implication morale où survient la dimension intrinsèquement temporelle de la personnalité. Dès lors, l'adolescent perçoit une exigence à dépasser la condition infantile qui le disposait à un certain rapport au monde, il entend alors devoir mûrir, il projette et désire grandir. Le franchissement de cette ligne de démarcation entre le monde proximal familier et le domaine public dessine donc un arc expérientiel remarquable à l'adolescence. Cet arc est intensément coloré par la prégnance nouvelle du domaine 
public. La faillibilité et l'affermissement progressif du moi, largement inexpérimenté et hésitant lors du franchissement de cette ligne, deviennent alors l'espace productif de compréhension de l'idée même d'adolescence.

Pour bien ouvrir cet angle de réflexion depuis le monde de perception des adolescents, il a fallu réajuster un champ notionnel et une langue descriptive permettant de développer une analyse fidèle à la manière dont la réalité du domaine public vient frapper le quotidien de l'adolescence. Ce réajustement prend appui sur une approche attentive aux connotations affectives qui accompagnent les dynamiques variées de l'affirmation de soi en public et tire profit d'une enquête récente (Breviglieri \& Cicchelli 2007) ainsi que de travaux ethnographiques évoqués au fil du texte.

\section{Régions ludiques et spatialités d'implication morale}

T es analyses sociologiques conduites sur l'enfance reposent sur un socle constitué de deux présuppositions anthropologiques : d'une part il y a, entre l'état d'enfant et l'âge adulte, une différence de nature dans l'être-ensemble avec d'autres ; d'autre part, l'enfance est d'emblée confrontée à un possible accroissement d'envergure (dirigé vers les pôles de la maturité et de la grandeur) qui tend à l'extraire de sa propre condition (l'enfant grandissant et mûrissant tend précisément à quitter l'enfance). La maturité et la grandeur indiquent la direction d'un état canonique relatif à l'âge adulte. La maturité se vise autonome et responsable au plan individuel face au souci des conséquences publiques ou privées de l'action ou de son omission (Pattaroni 2005). La grandeur correspond à la perspective d'une reconnaissance publique et légitime de la personne (Boltanski \& Thévenot 1991). Tout domaine public fonde et organise une diversité de mondes communs où les formes légitimes de la grandeur sont plurielles et demandent l'assurance d'une maturité pour être assumées en personne. Les épreuves publiques, à partir desquelles s'atteste la qualification publique de la personne, font ainsi ressentir le double axe normatif de la grandeur et de la maturité. C'est en ce sens qu'est évoquée ici une spatialité d'implication morale propre au domaine public.

L'enfant dispose initialement d'une spatialité naturellement topologique où il met en œuvre des activités manipulatrices en fonction d'une appréhension sensori-motrice de l'espace alentour appréhendé essentiellement comme des régions ludiques. Sa croissance et sa maturité requièrent et impliquent un certain itinéraire perceptif et cognitif qui l'invite toutefois à appréhender différemment cet espace (Piaget \& Inhelder 1948, de Saint-Aubert 2001). Cet itinéraire aboutit au stade où la spatialité d'implication de la personne peut être à la fois enveloppée d'un tissu conventionnel moralisé et enchevêtrée dans des histoires de texture variable. À ce stade, l'espace s'apparente alors à un domaine public disposé à 
accueillir une communauté. La question du rapport dynamique et pluralisé à l'espace se pose alors dans les termes d'une "pragmatique des régimes d'engagement" (Thévenot 2006). Les personnes font plus que structurer l'espace de manière topologique comme dans leur enfance, elles frayent des voies d'engagement sur des registres plus ou moins familiers ou collectifs et en suivant le fil d'histoires plus ou moins accaparantes. Cette dimension complexe de l'engagement dans le monde suppose une capacité de la personne à savoir composer ce que Thévenot désigne comme trois manières distinctes de s'engager dans son entourage, humain ou non (Thévenot 2006). À un régime d'engagement dans le familier où la personne trouve l'assurance d'un bienfait intime, s'ajoute un régime d'engagement "par le plan" par lequel se garantit un projet individuel bien intentionné (Thévenot 2006, 113-130) ; puis enfin un régime d'engagement par la justification permettant de certifier des convictions relatives à des biens communs. L'appréciation du monde (successivement en termes de propriétés familières, fonctionnelles et conventionnelles) reflète une diversité possible de points de vue, tandis que la capacité personnelle à composer avec les différents régimes d'engagements, "l'action au pluriel", témoigne des exigences de flexibilité et de pluralité auxquelles la modernité rend sensible, que cela concerne la sphère du travail, celle de la famille ou celle de la société civile.

\section{Espaces intercalaires}

'est en partie la composition et la maîtrise pragmatique de ces régimes d'engagement qui sont mises en jeu dans l'apprentissage des grammaires du domaine public qui se déplient à l'adolescence. L'enjeu, indiqué par les axes normatifs de la grandeur et de la maturité, est bien alors la participation à un agir en commun s'affirmant sur le domaine public. Comment rendre compte de cet apprentissage qui représente une composante essentielle de l'arc des expériences majeures de l'adolescent ? C'est ici que l'espace intercalé entre les régions ludiques de l'enfance et le domaine public se révèle important à questionner. Il permet de penser l'équilibre dynamique que trouve ou non la personne dans ses manières de s'engager dans le monde, entre la localité des régions ludiques du monde familier auxquelles l'adolescent reste attaché et la dimension généralisante du domaine public où il projette de s'affirmer. La délimitation notionnelle d'espace intercalaire, semble la prémisse pour comprendre, d'un point de vue pragmatique, le stade hésitant du "moi dubitatif" de l'adolescence. Elle permet de faire apparaître un geste hésitant qui esquisse une direction, ébauche un trajet et finit par représenter un "geste cheminatoire" (de Certeau 1990).

L'espace intercalaire représente un champ de gravitation pour un ensemble d'activités diverses où s'enclenche un apprentissage des modes de fonctionnement 
du domaine public. Ces activités, d'une part émergent du monde familier et proximal en cherchant, plutôt qu'une pleine séparation, à le tenir à distance relative (disons, à portée de vue ou de main); d'autre part s'approchent du domaine public en y quêtant un contact plutôt qu'une véritable immersion (le plaçant, lui aussi, à portée de vue ou de main). L'espace intercalaire donne l'occasion d'un affermissement graduel de la capacité du moi à s'ouvrir au domaine public. Il s'y renforce l'assurance de pouvoir se diriger en propre vers ce domaine, tout en pouvant (continuer de) compter sur le monde proximal et familier. La dynamique de cet affermissement du moi repose en partie sur une dialectique du désir et du besoin. Lorsque l'aspiration à l'accroissement d'envergure (pointé normativement par l'âge adulte) est ébranlée par les sentiments vertigineux d'avoir visé trop haut ou de ne pas être à la hauteur, le besoin d'un appui dans le monde proximal et familier se fait sentir, jusqu'à ce qu'il conduise à son tour au sentiment d'étouffement et au désir de libération de nouvelles aspirations.

\section{Recoins, cachettes, repaires}

T a forme empirique de l'espace intercalaire manifeste cette tentative de com_ position pragmatique. S'y affirment tant l'architecture du monde familier, où l'espace incline à être accommodé et domestiqué, que l'influence du domaine public qui espace, qui secrète de l'espacement, qui "esplace" selon l'expression de Goetz (2001). Les espaces intercalaires sont situés aux seuils, aux jointures, aux lignes de scission, partout où des mouvements d'oscillation sont facilités. Ils génèrent et entretiennent essentiellement des relations de voisinage ou de contiguité. Leur tension fondatrice suscite l'aménagement d'une ouverture vers l'horizon d'un domaine public rendu observable et "manipulable". On y édifie des parcelles habitables temporairement et qui tendent à déborder sur ce même domaine public. S'y déploient la recherche et l'affection pour les cachettes : coins, recoins, avancées, brèches, trouées, partout où l'on peut se replier sans perdre de vue les mondes communs et se tenir en retrait sans annuler la possibilité de la rencontre. On y décèle, enfin, plutôt qu'un espace divisé de façon binaire entre privé et public, un "chiasme intime-dehors" fondé sur un certain "consentement à la différence" (Salignon 1996). L'analyse topographique des lieux de rassemblement adolescents peut confirmer d'ailleurs cette idée d'espace intercalaire et de relation de contiguïté : ils se retrouvent le plus souvent devant le lycée et dans les cafés les plus voisins, dans le hall d'entrée de l'immeuble ou dans la cage d'escalier, aux abords du village ou à la lisière des places publiques les plus fréquentées, bref, à mi-chemin entre le chez-soi où réside encore le noyau de la dépendance infantile et les institutions socio-éducatives où se disséminent les premiers espaces probatoires du domaine public (Boissonade 2001, Jarvin \& Hachimi 2007, Kinzi 2007). 
Ces espaces intercalaires se caractérisent aussi par le flou de leurs lignes de démarcation. Un flou propice au jeu, au déploiement de l'exploration et de l'imaginaire, mais qui met aussi inlassablement en question la dimension de la confiance et de la précarité. Les analyses urbaines attentives à une pragmatique de l'usage de l'espace public, en décrivant les déambulations dans la rue ou l'occupation de places publiques, ont fréquemment approché la nature composite et les résonances affectives des espaces intercalaires. "Le monde des grandes métropoles en formation”, écrit Joseph $(1984,79)$, “est un tissu dont l'élasticité est indéterminée et qui demande qu'on dispose d'une langue nouvelle, la langue des intervalles". Dans la plupart de ces analyses, l'observation donne une place remarquable aux activités des adolescents. À l'élasticité indéterminée de l'espace intercalaire, qui correspond à un "certain quotient d'ouverture et de fermeture selon le réglage singulier instauré entre extérieur et intérieur” (Goetz 2001, 107), répond une visibilité intermittente où l'enjeu de la parution publique de l'adolescent appelle des tonalités émotionnelles fortes et passagères. Les jeunes ont un usage habitué des recoins sensiblement délabrés de certaines places publiques de la ville de Caracas, espaces à la fois intrigants et dérangeants, permettant une quiétude relative mais indiquant, simultanément, le rassemblement d'une certaine marginalité capable de soulever la méfiance du passant ordinaire (García Sánchez 2006). Dris (2001), enquêtant sur la mobilité dans les espaces publics d'Alger, signale la profusion d'espaces où peut s'installer une logique du flou qui permet aux jeunes de s'accommoder, dans l'espace public urbain, du maintien des valeurs familiales. Ainsi, des marchands de vin ont installé des rideaux, aménagé des chicanes, bâti des morceaux d'espace où on peut ne pas être vu pour "se faire oublier" et "transgresser ces valeurs" (180). Considérée dans le climat moral ambiant comme un lieu de débauche, la rue est pour ses usagers un espace d'opportunités, de trafic, de drague, où se marquent différents seuils, différents paliers et différents registres d'accessibilité à l'espace public (Dris 2001, 177-241). Les jeunes affectionnent le quartier ("houma") en tant qu'il figure un "espace tampon entre les espaces publics de la centralité et les espaces domestiques", mais aussi un premier accès aux grands axes de la métropole, un "support aux échanges collectifs" et un balisage identitaire sur fond d'activités familières capables de rendre saillants les traits de l'étranger qui s'y aventure (Dris 2001, 180).

\section{Esquives, combines}

T es cachettes et repaires offerts par les espaces intercalaires ont un caractère matriciel concernant le registre des actions reflétant un art du décalage. À cet égard, se détachent notamment l'esquive et la combine. La présence chancelante du moi dubitatif de l'adolescent sur le domaine public ne doit parfois son maintien 
qu'au prix de l'esquive de certaines épreuves et plus largement d'un certain art à combiner les régimes d'engagement dans le monde sans ébranler son ordre commun. L'esquive et la combine plongent encore leurs racines créatives dans la production ludique d'espaces potentiels. Mais elles disposent en plus d'une sagacité, d'un esprit d'à propos, d'une science du "kairos" (de la bonne occasion) qui les met au contact du domaine public dans un mouvement d'ajustement dynamique et de transposition habile. Elles en retirent un certain brio auprès de ceux qui partagent un même espace intercalaire, une même position décalée, une même orientation oscillante. Il y a des champions de l'esquive et des rois de la combine. Mais du point de vue des repères institutionnels et des axes de coordonnées du domaine public, elles restent relatives et inqualifiables. L'esquive et la combine sont tour à tour irritantes et provocantes en s'installant dans l'espace commun par un geste flou et décalé dont l'intention demeure partiellement insaisissable pour un regard extérieur. Elles n'ont pas une pleine légitimité sur le domaine public, paraissant même avancer avec la lâcheté de qui fuit les épreuves. Mais qui les craint? Qui trompent-elles ? Ni l'esquive, ni la combine ne lèvent l'ombre d'une menace sérieuse sur l'institution du domaine public, elles ne font qu' "inventer du quotidien" (de Certeau 1990). L'esquive n'a pas la gravité et la caisse de résonance de la défection qui vaut comme une véritable sanction publique. La combine produit un assemblage astucieux et intéressé mais qui n'a pas l'ambition des grandes manœuvres briguant un pouvoir sur le domaine public. Elles ne sont jamais vraiment menaçantes bien qu'elles soient déroutantes, car elles n'avancent que sur le périmètre d'action permis et restreint par la précarité.

\section{Étincellement}

E nvisagé comme chiasme, l'espace intercalaire voit s'entrelacer les résonateurs affectifs de la séparation du monde proximal habité, de la découverte et ceux de la percée sur le domaine public. Aux résonateurs, positifs de l'exaltation et de la curiosité et négatifs de l'anxiété et de la crainte, correspondent les mouvements maladroits qui conduisent à des fautes de tact : l'irruption brutale dans la coprésence (indiquant un défaut de réserve) et le retrait outré (qui révèle un excès de retenue). Ces mouvements restent apparentés aux pulsions motrices primordiales de l'enfance, bondir et se blottir. Au bondissement, à l'irruption et à l'ivresse de l'excentricité qu'un même ressort semble animer, correspond la modalité phénoménale d'apparition valeureuse sur le domaine public de l'étincellement. La tonalité affective de l'étincellement surprend son public et déclenche l'impression admirative qu'un geste brillant s'est soudainement imposé à l'ordre du monde commun. De telles dynamiques de reconnaissance des virtuosités techniques sont, par exemple, scrupuleusement étudiées dans une ethnographie des arènes publiques du jeu informatique (Auray 2000). Dans l'étincellement, la précarité du moi 
hésitant a trouvé une combine pour pénétrer de manière éphémère le domaine public dans la direction d'une reconnaissance. La visibilité intermittente de celle ou celui qui se tient activement au seuil du domaine public, sur des espaces intercalaires, invoque un mérite qui déjà tient lieu de promesse faite à autrui. Par exemple, en esquivant brillamment par la parole certaines épreuves, la personne aura su convaincre une audience de son éloquence et mettre en perspective les potentiels dont recèle cette dernière. Mais l'étincellement a aussi le défaut d'une promesse dont rien ne garantit pleinement la fiabilité. Il fait reluire des potentialités (ce dont on peut être capable) sans témoigner d'un réel pouvoir (ce dont on est capable).

Le coup d'éclat de l'action étincelante dispose ainsi de la faculté d'aveugler le public sur la réelle consistance des capacités engagées. De là, s'aperçoit le confort des espaces intercalaires : l'intermittence de la parution, la possibilité du retrait, l'art du décalage permettent de pouvoir ne pas rendre de comptes sur le domaine public. L'esprit d'à propos peut bien refuser de se colmater avec un “esprit public" (Cotterau 1992). Il est tentant de s'illustrer sur le mode fugitif et passager de l'étincellement pour éviter l'axe normatif de l'agir responsable et mûr qui, pour sa part, est déjà aux prises avec la durée d'un temps long. Ce bonheur de se sentir sans réelle consistance temporelle, de ne produire aucune attente envers soi est aussi une manière de laisser grande ouverte la liberté du seul coup d'éclat. Mais en voyant se démultiplier autour de lui les espaces probatoires et leurs attentes en termes de conséquences morales à assumer, l'adolescent voit en quelque sorte s'évanouir une à une ces possibilités heureuses. Parallèlement, l'étincellement laisse pointer ses propres limites sur la durée. En se répétant, elle devient un flux constant de lumière, elle quitte l'habit du simple étincellement. Avec sa réitération, surgit la pression des attentes : le coup d'éclat ne surviendra plus aussi librement car la manifestation régulière des habiletés a fait éclore la reconnaissance d'une certaine grandeur en affirmant dans la durée un style personnel. De plus, cette répétition tend à convertir le geste spontané habile en fait social régulier : il paraît alors comme une habitude pratique face à un ordre normatif. Le coup d'éclat peut alors bien devenir sous cet angle une "mauvaise habitude” de joueur ou de provocateur dont l'écueil le plus grave est repéré aujourd'hui par une littérature psychosociologique sous les traits pathologiques des addictions de l'adolescent (Valleur \& Matysiak 2004).

\section{Embrouilles}

T orsque l'irruption soudaine sur le domaine public est commandée par une intention d'y briller en produisant un coup d'éclat, elle soulève une tension dramaturgique éventuellement renforcée par un faisceau polémologique. Le domaine public peut alors être envisagé sous l'aspect et les qualités spécifiques de 
l'arène publique (Cefaï 2002). Comme on vient de le voir, les espaces intercalaires lorgnent vers le domaine public, débordent et empiètent sur lui. Les situations d'arènes s'inscrivent bien dans la structure équivoque des espaces intercalaires. Elles prennent consistance au cœur des régions ludiques à travers les jeux de la provocation qui captivent et font frémir l'assemblée. L'arène surgit ici au cœur de simples rapports entre proches. La notion de rapport évoque toutefois l'idée que, au-delà d'une apparente banalité du quotidien, se dégagent des événements et des éléments dont on peut se rendre témoin et qui se rapportent sous forme de narrations anecdotiques, ce qui, avec l'appui du langage, les fait souvent gagner en généralité. Tel est le cas de l'embrouille où l'attribution arbitraire de différences entre soi, qui comporte le plus souvent une pointe d'offense, s'élève dans une confusion relative aux termes mêmes du rapport. Typiquement, pour commencer, celui qui va vers l'autre "cherche l'embrouille" en affirmant que cet autre "le cherche". L'embrouille constitue une prémisse d'arène publique. D'une part, elle suscite une tension attentionnelle, elle attire l'attention en dilatant le trouble de la situation. D'autre part, dépourvue d'orientation distincte, elle suscite un désir de clarification et précipite la convocation d'un tiers susceptible d'apporter un regard objectif. L'embrouille correspond en un sens au brouillage des lignes de démarcation séparant les "régimes d'engagement dans le monde" (Thévenot 2006). L'“embrouilleur" est a priori difficile à cerner : les ressorts intimes de son comportement restent obscurs, ses justifications sont confuses, son action ne semble pas concorder avec ses intentions. Il s'attarde sur des parcelles de monde familier car ses proches sont finalement les plus concernés par l'enchevêtrement de ses histoires intimes et publiques. Mais si le vecteur tensoriel de l'embrouille oriente vers la constitution d'une arène où se déploie la dimension évaluative de l'épreuve publique, ce sont alors généralement les proches qui, soudain, édifient de l'espace entre eux, jouant eux-mêmes le rôle des tiers en donnant plus ou moins l'impression de représenter un public. Tout en n'atteignant pas ici la légitimité instituée des espaces probatoires, l'arène acquiert l'intensité émotionnelle d'une véritable épreuve en public à mesure que les proches prennent au sérieux et gagnent en crédibilité dans leur rôle de tiers.

Une ethnographie réalisée dans la cité des Quatre-Mille à La Courneuve approche très précisément la manière dont une "sociabilité de voisinage" entre adolescents, "à l'écart et hors du regard des adultes”, peut assez largement reposer sur une structure de rapports contaminée par les exigences pragmatiques de l'arène (Lepoutre 2001). Partant de l'observation de l'attachement résidentiel des jeunes, elle analyse comment s'immiscent continûment dans les contacts quotidiens des provocations ou des embrouilles étroitement mêlées aux liens de proximité et toujours capables de les renforcer. C'est en faisant varier sensiblement la gravité de l'atteinte portée par ces provocations (la vanne, l'insulte, l'offense d'un côté, les échauffourées, la bagarre, les rixes de l'autre côté) que se gère graduellement et 
subtilement l'excitation relative à la publicisation des rapports qui culminent précisément au stade de l'arène publique. Le flou des lignes de démarcation tient au fait que, des "joutes oratoires" aux "querelles d'honneur", le jeu est partout et s'entremêle constamment à un "habitus agonistique" (Lepoutre 2001, 173-204 et 375-397). C'est enfin à ce stade que s'aperçoit, sur ces espaces intercalaires, la contiguité au domaine public : les sphères de moralité sont appelées pour trancher sur la qualité des participants et pour convoquer éventuellement des formes de réparations. Mais la limitation et la territorialisation strictes de cette culture des rues adolescente tiennent aussi à ce dernier point : loin de se confondre avec la pluralité constitutive du domaine public et de la société civile, les convictions morales sont restreintes à la dimension de l'honneur et le système de réparation à une justice vindicatoire. L'étroitesse d'un tel monde moral laisse entendre le pourquoi de la brièveté du temps passé par les adolescents au cœur de la culture des rues dont parle Lepoutre. Bien vite, ils prétendent à la légitimité dans d'autres sphères d'activité, ils placent leurs ambitions ailleurs et se retrouvent face à la pluralité des mondes communs du domaine public. Ainsi, l'étincellement dans les arènes publiques de la culture des rues s'évanouit à l'appel d'une maturité requise par la dynamique de l'insertion professionnelle (où émergent des "préoccupations en termes de projets personnels") et dès lors que pointe l'horizon d'une autre grandeur quand "la curiosité se déplace à l'extérieur du quartier" (Lepoutre 2001).

\section{Bravoure}

T 'arène publique, où se produit l'étincellement du geste habile, a la temporaL lité de la fabrication de l'événement et non, comme le domaine public, la temporalité des institutions qui la fondent. Elle a aussi la qualité d'un "espace agonistique où chacun cherche à affirmer sa grandeur" en faisant valoir un "ethos de la virtuosité". Cette approche de l'arène publique prend en compte les travaux de Cefaï (2002) sur la contribution des arènes publiques aux dynamiques d'élaboration de cultures publiques et ceux de Dodier (1995) sur les arènes de l'habileté technique qui recadrent l'arène dans sa dimension originelle dramaturgique. Le principe de l'arène publique recèle une dynamique émotionnelle qui s'instaure dans une situation trouble d'où émerge une tension dramatique liée à la nature du défi et qui, pour finir, vacille de manière paroxystique au moment clôturant du jugement. Si le trouble peut être, au départ, insensible, il reste que chaque provocation faite dans l'arène accroît la densité émotionnelle, réveille de façon brutale des espaces chargés d'attentes, élargit l'assistance et assigne l'audience à une attention prononcée. Cet "épaississement tensoriel" est nécessaire à la préparation et à la réception de l'exploit (Gély 2000). Cette réception de l'exploit est aussi un accueil donné au monde proximal et familier qui siège encore dans les 
espaces intercalaires. L'habileté qui se déploie en virtuosité reflète d'abord la manière dont le monde familier s'est rendu disponible à l'usage et se trouve être à la main de la personne (Breviglieri 1999) ; en quelque façon, "le virtuose peut agir grâce à des 'tours de mains', à des habitudes acquises depuis l'enfance” (Dodier 1995, 224). La métis, dont est doté le virtuose, lui permet de transposer ces tours de mains, acquis dans l'usage familier d'un monde proximal, à l'échelle du public. Elle repose sur un savoir de la situation (kairos) qui se déplace sans relâche dans une dimension spatio-temporelle : du point de contact opportun trouvé dans le milieu physique au moment propice.

Des travaux étymologiques ont montré que, à l'image du talon d'Achille, le kairos, dans son acception première, désigne l'endroit vulnérable du corps, là où la blessure s'avère être fatale (Schuhl 1962, Trédé 1984). L'occasion propice résulte de la perception des lieux décisifs sur lesquels l'homme à la métis s'appuie pour renverser la situation en sa faveur. Le sens local de kairos a néanmoins évolué vers un sens plus temporel. Ce développement, où s'affirme le rôle du logos dans l'action, a été particulièrement mis à profit par les sophistes à travers les techniques argumentatives permettant de remporter les joutes verbales. En appelant la dimension temporelle, la métis du virtuose est amenée à considérer son influence sur l'audience et donc à estimer les conséquences de son action auprès d'un public. Mais l'arène publique ne requiert pas simplement la clairvoyance des ruses de l'approximation, elle réclame aussi le courage qui pousse à la bravoure au moment où l'espace du doute pourrait inhiber toute initiative : un courage d'apparaître en public et de répondre de ce que cela suppose. S'accomplir dans la virtuosité regarde alors la nature phénoménale du politique où se pose la question du choix du monde où apparaître et vivre ensemble (Tassin 1996, 88). Il s'agit, comme le souligne Arendt, "de quitter la sécurité protectrice de nos quatre murs et d'entrer dans le domaine public, cela demande du courage, (...) parce qu'en politique, ce n'est pas la vie mais le monde qui est en jeu" (Arendt 1995, 203).

\section{Retranchement (derrière les proches et sur soi-même)}

T 'aspect dramaturgique des arènes publiques ne tient pas simplement à l'étincellement du virtuose et à la manière dont il interpelle son public. Il repose aussi, nécessairement même, sur la tragédie de l'échec. Sur le fond polémologique de l'arène, l'échec n'est pas seulement le produit normal du tâtonnement exploratoire, c'est une défaite capable d'affliger l'estime de soi. Erikson (1972) est attentif à l'angoisse d'être poussé dans des activités où l'adolescent pourrait se sentir exposé au ridicule ; sa volonté, dit-il, est particulièrement chancelante dès lors que pointe l'horizon du sentiment de honte (134). L'appréhension des arènes publiques et, plus loin, des espaces probatoires du domaine public, emmène alors la 
tonalité de la trouille au-devant de la crainte de mal faire et de la "peur du ridicule". Cette conception dramatique de la parution en public induit une tension sensible extrêmement vive où la moindre maladresse en public peut convoquer l'impression de "mourir de honte". Lorsque l'affirmation de soi n'est pas encore consolidée dans une reconnaissance publique, l'échec face à un auditoire révèle d'emblée, sous les failles ouvertes, une absence de fondation pour se montrer capable d'être autonome dans l'épreuve. En se produisant à l'adolescence, où les axes normatifs de la grandeur et de la maturité soulèvent le désir de croissance et l'espoir d'élévation, cet aveu de faiblesse joue sur le même front néfaste que la violence de l'infantilisation. L'adolescent s'y dévoile contre son gré sous les traits fragiles et maladroits de l'enfance. Sous cet angle, le domaine public reprend son visage de monde hostile à partir duquel s'amorce un retrait vers le monde familier et proximal à travers lequel on perçoit la pulsion motrice du blotissement contre le proche. À l'étincellement s'oppose la modalité phénoménale d'apparition malheureuse sur le domaine public du retranchement derrière le proche. Ce retranchement n'évoque pas ici le départ ou la défection du domaine public et le besoin des fonctions de refuge du monde proximal, mais le lien de causalité entre l'échec ou la défaite subie et l'érection d'une fortification autour du soi, d'une carapace censée protéger et couper de ce domaine négativement perçu.

La dimension radicalement négative du retranchement est rappelée, en des termes pathologiques, par la psychanalyse ou la psychiatrie. Concernant l'adolescence, Erikson interroge la gestion identitaire des épreuves traumatisantes et leurs conséquences en termes de détachement de la réalité ou, autrement dit, de "désinscription" du cours du monde. L'adolescent évoque alors le problème majeur de l'attardement comme conséquence de son retranchement durable d'une sphère d'expérience en public (Erikson 1972, 188-198). Erikson convient que l'adolescent, en sortant ou en refusant de s'inscrire dans le cours du monde, en "dissolvant la perspective temporelle", s'expose à de graves confusions identitaires qu'il illustre par le cas d'adolescences retardées et prolongées (Erikson 1972, 177). À l'effort conséquent pour bénéficier du "sentiment véritable de réalité dans des rôles acceptables", qui correspond au fait de s'inscrire dans le cours du monde, l'adolescent préfère parfois la production d"'identités négatives" déconnectée de toute épreuve de réalité. D'un côté il tend alors à refuser toute forme de vivre ensemble, allant même jusqu'à dédaigner son propre monde proximal familier et à s'isoler dans un retranchement sur soi-même. De l'autre, il incline à constituer des espaces coupés de la réalité du monde, depuis "la nécessité de trouver ou de défendre un petit coin bien à soi" jusqu'à la quête d'une "aire sociale totalement marginalisée" (Erikson 1972, 183). 


\section{Pédagogies libérales de l'empiétement}

T a lecture pathologique du mouvement de retranchement, entendu comme une liquidation identitaire et la conséquence d'effets pervers relatifs à des occurrences traumatiques, doit être nuancée dès qu'on revient à l'idée que les espaces intercalaires configurent aussi des mouvements constructifs et des dynamiques d'apprentissage. On a avancé l'idée que ces espaces intercalaires, où résonnent encore puissamment les régions ludiques de l'enfance, tendent à empiéter et à déborder sur le domaine public. L'arc des expériences concernées est vaste et quelques aspects ont été évoqués sous l'angle de leur potentiel provocateur : tentative de séduction, recherche d'embrouille, incursion sur des territoires interdits, détournement de l'ordre bâti et des conventions instituées par et pour le domaine public, etc. L'empiétement ou l'intrusion représentent des gestes offensifs remarquables dans la dynamique d'apprentissage des grammaires morales du domaine public. Ils possèdent une tonalité provocatrice qui irrite et appelle au sentiment d'excès de proximité, d'appropriation illégitime d'un espace ou de violation d'un territoire privé (Breviglieri 2007). Ces gestes soulèvent des réactions vives fondées sur une indignation qui se rattache à la distinction légitime du mien et du tien, et qui permet l'identification concrète de la sphère privée. Or celle-ci est culturellement envisagée, dans la tradition intellectuelle libérale et démocratique, d'une part comme une condition de préservation de l'autonomie individuelle et, d'autre part, et non sans liens, comme entretenant un lien mécanique de réciprocité avec le domaine public. La sphère privée se rend tangible dans l'empiétement : elle se donne alors comme un vécu apparaissant qui soulève une indignation morale et révèle des points de tensions particulièrement sensibles. À ce point de tension, c'est tant la vigilance aux dynamiques du domaine public, que leur lien ténu aux axes normatifs de la maturité et de la grandeur qui sont appelés.

De ce point de vue, les espaces intercalaires instruisent activement et mettent en lumière ces gestes cheminatoires qui avancent avec tact vers le domaine public sans s'échouer sur son seuil. Cette pédagogie de l'empiétement joue en réalité dans un double sens. L'apprentissage ne concerne pas seulement le réglage progressif de l'élan physique et intentionnel qui meut les gestes d'empiétement et d'intrusion, il va aussi, en sens opposé, vers la maîtrise d'une protection du soi en public. Ainsi l'esquive et la combine se retrouvent placées sur une polarité entre deux gestes de nature différente mais convergeant vers l'idée qu'ils déploient, chacun à leur manière, un tact face à autrui. La combine relève ainsi d'un geste offensif (toujours réalisé au risque de l'intrusion et de la violence de l'irruption), alors que l'esquive se rapporte à un geste défensif (effectué au risque du retrait outré, deuxième modalité de la faute de tact identifiée précédemment). Le geste de retranchement, qui tend à se replier pour trouver une protection peut ainsi, par symétrie, convoquer une même sphère de moralité que le geste d'empiétement auquel il apporte une réponse prudente. Il s'apparente alors à un geste de conso- 
lidation de la sphère privée et de mise en garde face aux empiétements d'autrui. Ce type de vigilance fait émerger l'architecture normative du domaine public de tradition libérale et démocratique où règne une volonté de libre mobilité des hommes et de leurs biens et où l'empiétement appelle une résistance à l'occupant car, une fois amorcé, il semble pousser inexorablement à l'absorption totale du sujet.

\section{Carapaces}

L e discernement des qualités publiques et privées de l'action et des espaces, que l'enfant maîtrise mal, suscite un net souci chez l'adolescent car il représente une modalité prégnante de l'acquisition de la maturité. La syntaxe de l'agir en commun repose en partie sur la délimitation de ces deux sphères qui s'identifie de manière basique par une clôture assurant la protection d'une sphère privée et offrant l'assurance que nul gouvernement ne peut s'exercer de manière coercitive. Cette clôture permet notamment de préserver les choses privées de l'espace probatoire du domaine public. Elle constitue aussi une manière d'apercevoir ce que coûte l'accès au domaine public. Ce coût a été envisagé précédemment en termes de courage avancé par celui qui franchit le seuil du domaine public. Il s'aperçoit ici à partir de ce que procure le fait de s'épargner une parution en public. Certaines analyses portant sur l'espace physique personnel et sur le jardin secret de l'adolescent donnent très concrètement à penser la manière dont il apprend à constituer une distance de prévention et un espace de protection vis-à-vis de son entourage. Son étant-sur-la-défensive s'emploie à préserver l'inviolabilité d'un "territoire de l'intimité personnelle" de la "menace" que représentent alors la société comme la famille (Neuburger 2000). C'est en exprimant les malaises relatifs à l'intrusion dans ce territoire (dérangement dans le temps, inquiétude relative à l'empiétement de l'espace, jalousie de sa liberté et de ses propres biens...) que se consolide physiquement et symboliquement une carapace protectrice. C'est aussi par ce biais que se fortifie le sentiment positif du territoire privé qui, lorsque s'introduit l'idée de la propriété légitime, appelle l'entrée en scène du droit positif. Winnicott (1994) mentionne la fréquence du vol chez les adolescents dont on peut dire qu'elle converge aussi vers l'apprentissage du rôle structurel de la propriété privée sur le domaine public. Il reste que la carapace, derrière laquelle la personne craintive peut se retrancher en se coupant radicalement du monde, est un cas empirique limite de l'espace intercalaire. L'épaisseur de la carapace peut bien empêcher la contiguïté avec le monde extérieur. Le mouvement d'oscillation, où la sortie en public s'assure de manière intermittente, s'est peut-être alors déjà figé dans un ferme repli, dans une absence d'ouverture à l'autre, et pour finir dans une carence absolue d'apprentissage.

Dans un certain sens, l'adolescent s'achemine en étranger sur le domaine public. Il partage avec l'étranger venant d'une autre communauté le fait qu'en 
accédant à un espace fondé pour l'agir en commun, il doit se rendre capable d'encaisser des chocs imprévus sans ruiner sa disposition à la mobilité. La carapace représente alors une capacité maximale à l'“encaissement du choc des épreuves" dont parle Stavo-Debauge (2007). Mais l'étrangéité de l'adolescent est particulière. Il ne vient pas à la rencontre d'une autre communauté, il provient de l'enfance et des privilèges que lui accordent généralement tant ses proches que l'humanité en général. Ce qu'il apprend, c'est à convertir, à petits pas, son monde familier ambiant dans une spatialité d'implication morale et politique, puis à inscrire temporellement son implication dans le cours du monde. Il élargit son horizon à condition qu'il sente venir et qu'il commence à assumer les prérogatives primordiales de l'agir en commun. En cela, faire partie d'un monde commun demande moins l'assurance d'en être ou d'y être que l'incertitude de ce qu'on lui doit exactement. Il n'est donc pas seulement question pour l'adolescent de se prémunir de l'arête tranchante des espaces probatoires, mais aussi de ne pas totalement s'en protéger (ou d'en être protégé). Car il pourrait alors se rendre insensible aux résonateurs affectifs qui enclenchent les dynamiques d'apprentissage requises à l'approche du domaine public dont il n'avait pas l'idée au stade de la dépendance infantile. C'est pourtant sur cette base expérientielle et affective primordiale que s'exerce et s'instruit un savoir-faire consistant à s'inscrire graduellement et à s'affirmer avec tact sur ce domaine public.

\section{Références bibliographiques}

ARENDT H. 1983 Condition de l'homme moderne, Paris, Calmann-Lévy

ARENDT H. 1995 Qu'est-ce que la liberté ?, in La crise de la culture, Paris, Gallimard

AURAY N. 2000 Politique de l'informatique et de l'information. Les pionniers de la nouvelle frontière électronique, Thèse de doctorat, Paris, EHESS

BOISSONADE J. 2001 "Agrégations juvéniles et dynamiques du proche", Les Annales de la Recherche Urbaine-90

BOLTANSKI L. \& THÉVENOT L. 1991 De la justification, Paris, Gallimard

BREVIGLIERI M. 1999 L'usage et l'habiter. Contribution à une sociologie de la proximité. Thèse de doctorat, Paris, EHESS

BREVIGLIERI M. 2007 “L'insupportable. L'excès de proximité, l'atteinte à l'autonomie et le sentiment de violation du privé", in Breviglieri, M., Lafaye, C. \& Trom, D., Sens de la justice, sens critique, Paris, Economica

BREVIGLIERI M. \& CICCHELLI V. 2007 Adolescences méditerranéennes. L'espace public à petit pas, Paris, L'Harmattan

BREVIGLIERI M. \& STAVO-DEBAUGE J. 2007 Sous les conventions. Accompagnement social à l'insertion : entre sollicitude et sollicitation, in Eymard-Duvernay \& al., Conventions et Institutions : approfondissements théoriques et contributions au débat politique, Paris, La Découverte 
CEFAÏ D. 2002 Qu'est-ce qu'une arène publique ? Quelques pistes pour une approche pragmatiste, in Cefaï, D. \& Joseph, I. (dir.), L'héritage du pragmatisme. Conflits d'urbanité et épreuves de civisme, La Tour d'Aigues, Éditions de l'Aube

COTTERAU A. 1992 "Esprit public" et capacité de juger, in Cottereau A. \& Ladrière P. Pouvoir et légitimité. Figures de l'espace public, Raisons Pratiques-3

de CERTEAU M. 1990 L'invention du quotidien. 1. Arts de faire, Paris, Gallimard

DODIER N. 1995 Les Hommes et les Machines. La conscience collective dans les sociétés technicisées, Paris, Métailié

DRIS N. 2001 La ville mouvementée. Espace public, Centralité, mémoire urbaine à Alger, Paris, L'Harmattan

ERIKSON E.-H. 1972 Adolescence et crise. La quête identitaire, Paris, Flammarion

GARCÍA SÁNCHEZ P.-J. 2006 "Entre urbanité et ordre public. Une écologie de l'usage des places à Caracas", Espaces et Sociétés-125

GÉLY R. 2000 La genèse du sentir. Essai sur Merleau-Ponty, Bruxelles, Éditions Ousia

GOETZ B. 2001 La dislocation. Architecture et philosophie, Paris, Les Éditions de la Passion

HACHIMI-ALLAOUI M. \& JARVIN M. 2007 Les usages des cafés lycéens. Entre discussions intimes et exposition de soi, in Breviglieri M. \& Cicchelli V. Adolescences méditerranéennes. L'espace public à petit pas, Paris, L'Harmattan

JOSEPH I. 1984 Le passant considérable. Essai sur la dispersion de l'espace public, Paris, Librairie des Méridiens

KINZI A. 2007 La jeunesse en milieu rural kabyle et ses rapports conflictuels avec les institutions villageoises, in Breviglieri M. \& Cicchelli V. Adolescences méditerranéennes. L'espace public à petit pas, Paris, L'Harmattan

LEPOUTRE D. 2001 Coeur de banlieue. Codes, rites et langages, Paris, Odile Jacob

MAHFOUDH-DRAOUI D. \& MELLITI I. 2006 De la difficulté de grandir. Pour une sociologie de l'adolescence en Tunisie, Tunis, Centre de Publication Universitaire

MERLEAU-PONTY M. 1994 Phénoménologie de la perception, Paris, Gallimard

PATTARONI L. 2005 Politiques de la responsabilité. Promesses et limites d'un monde fondé sur l'autonomie, thèse de doctorat, Université de Genève et EHESS

NEUBURGER R. 2000 Les territoires de l'intime. L'individu, le couple, la famille, Paris, Odile Jacob

PIAGET J. \& INHELDER B. 1948 La représentation de l'espace chez l'enfant, PUF

SAINT-AUBERT E. de 2001 "Sources et sens de la topologie chez Merleau-Ponty", Alter-9

SALIGNON B.1996 Le seuil, un chiasme intime-dehors, in Le sens du lieu, Bruxelles, Éditions Ousia

SCHUHL P.-M. 1962 "De l'instant propice", Revue philosophique-CLII

STAVO-DEBAUGE J. 2007 Venir à la communauté, Pour une sociologie de l'hospitalité et de l'appartenance, thèse de doctorat, EHESS

TASSIN E. 1996 La question de l'apparence, in Politique et pensée. Colloque Hannah Arendt, Paris, Payot

THÉVENOT L. 2006 L'action au pluriel. Sociologie des régimes d'engagement, Paris, La Découverte

TRÉDÉ M. 1984 "Kairos : problèmes d'étymologie", Revue des Études grecques-XCVII

VALLEUR M. \& MATYSIAK J.-C. 2004 Les nouvelles formes d'addiction. L'amour, le sexe, les jeux vidéo, Paris, Flammarion

WINNICOTT D.-W. 1994 Déprivation et délinquance, Paris, Éditions Payot \& Rivages 